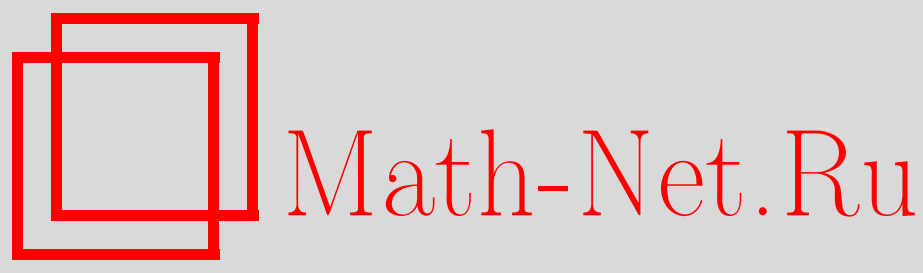

А. В. Зотов, Старшие аналоги условия унитарности для квантовых $R$-матриц, $T M \Phi, 2016$, том 189 , номер $2,176-$ 185

DOI: https://doi.org/10.4213/tmf9097

Использование Общероссийского математического портала Math-Net.Ru подразумевает, что вы прочитали и согласны с пользовательским соглашением http: //www . mathnet.ru/rus/agreement

Параметры загрузки:

IP : 35.174 .16 .151

26 апреля 2023 г., 16:25:42

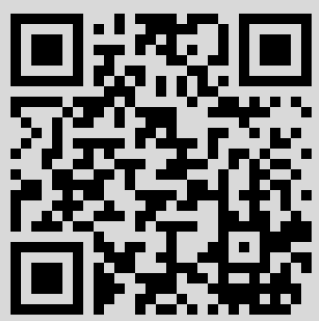




\title{
ФИЗИКА
}

Том 189, № 2

ноябрь, 2016

(C) 2016 г.

А. В. Зотов*

\section{СТАРШИЕ АНАЛОГИ УСЛОВИЯ УНИТАРНОСТИ ДЛЯ КВАНТОВЫХ $R$-МАТРИЦ}

\begin{abstract}
Выведено семейство тождеств $n$-го порядка для квантовых $R$-матриц типа Бакстера-Белавина в фундаментальном представлении. Набор тождеств включает условие унитарности как простейший случай (при $n=2)$. Тождество третьего порядка обеспечивает коммутативность связностей Книжника-Замолодчикова-Бернара. С другой стороны, оно же приводит к $R$-матричнозначным парам Лакса для классических интегрируемых систем типа Калоджеро. При выводе тождеств используется интерпретация квантовой $R$-матрицы как матричного обобщения функции Кронекера. Предложено доказательство тождеств старшего порядка для функций Кронекера, которое затем естественно обобщается на тождества для $R$-матриц.
\end{abstract}

Ключевые слова: классические интегрируемые системы, R-матричнозначные представления Лакса, дуальности.

DOI: $10.4213 / \operatorname{tmf} 9097$

\section{1. ВВЕДЕНИЕ}

Квантовая $G L(N, \mathbb{C})$ - $R$-матрица является решением квантового уравнения Янга-Бакстера

$$
R_{12}^{\hbar}\left(z_{1}, z_{2}\right) R_{13}^{\hbar}\left(z_{1}, z_{3}\right) R_{23}^{\hbar}\left(z_{2}, z_{3}\right)=R_{23}^{\hbar}\left(z_{2}, z_{3}\right) R_{13}^{\hbar}\left(z_{1}, z_{3}\right) R_{12}^{\hbar}\left(z_{1}, z_{2}\right) .
$$

В фундаментальном представлении $R$-матрица $R_{12}^{\hbar} \in \operatorname{Mat}(N, \mathbb{C})^{\otimes 2}$. При этом $R_{a b}^{\hbar}$ с $1 \leqslant a, b \leqslant n$ следует понимать как элемент пространства $\operatorname{Mat}(N, \mathbb{C})^{\otimes n}$, равный тождественному оператору во всех компонентах тензорного произведения, кроме компонент с номерами $a$ и $b$. Проекция на эти компоненты совпадает с $R_{12}^{\hbar}$. Рассматриваемые $R$-матрицы зависят только от разности спектральных параметров. Будем использовать обозначение

$$
R_{a b}^{\hbar}=R_{a b}^{\hbar}\left(z_{a}-z_{b}\right)
$$

Исследование выполнено за счет гранта Российского научного фонда (проект № 14-50-00005).

* Математический институт им. В. А. Стеклова РАН, Москва, Россия. E-mail: zotov@mi.ras.ru 
Класс $R$-матриц, с которыми мы будем работать, включает как простейший случай рациональную $R$-матрицу Янга [1]

$$
R_{12}^{\mathrm{Yang}}\left(z_{1}, z_{2}\right)=\frac{1 \otimes 1}{\hbar}+\frac{N P_{12}}{z_{1}-z_{2}}, \quad P_{12}=\sum_{i, j=1}^{N} E_{i j} \otimes E_{j i},
$$

а также ее деформации [2]-[4], тригонометрические $R$-матрицы [2], [5] и эллиптическую $R$-матрицу Белавина-Бакстера [6], [7].

Помимо (1.1) $R$-матрица удовлетворяет условию унитарности $R_{12}^{\hbar} R_{21}^{\hbar}=1 \otimes 1$. Мы будем использовать другую нормировку:

$$
R_{12}^{\hbar} R_{21}^{\hbar}=N^{2} \phi\left(N \hbar, z_{1}-z_{2}\right) \phi\left(N \hbar, z_{2}-z_{1}\right) 1 \otimes 1=N^{2}\left(\wp(N \hbar)-\wp\left(z_{1}-z_{2}\right)\right) 1 \otimes 1,
$$

где $\phi(\eta, z)$ - функция Кронекера [8]. В зависимости от выбора между рациональным, тригонометрическим и эллиптическим случаями она равна ${ }^{1)}$

$$
\phi(\eta, z)= \begin{cases}\frac{1}{\eta}+\frac{1}{z}, & \text { рациональный случай, } \\ \operatorname{cth} \eta+\operatorname{cth} z, & \text { тригонометрический случай, } \\ \frac{\vartheta^{\prime}(0) \vartheta(\eta+z)}{\vartheta(\eta) \vartheta(z)}, & \text { эллиптический случай. }\end{cases}
$$

Правая часть равенства (1.4) содержит функцию

$$
\wp(z)= \begin{cases}\frac{1}{z^{2}}, & \text { рациональный случай, } \\ \frac{1}{\operatorname{sh}^{2} z}, & \text { тригонометрический случай, }\end{cases}
$$

а в эллиптическом случае $\wp(z)$ совпадает с эллиптической ю-функцией Вейерштрасса. В соотношении (1.4) уже использовано тождество

$$
\phi(\eta, z) \phi(\eta,-z)=\wp(\eta)-\wp(z) .
$$

Как было обнаружено в работе [11] и позднее в [12], [9], $R$-матрицу Белавина можно рассматривать как матричное обобщение эллиптической функции Кронекера ${ }^{2}$. В частности, она удовлетворяет матричному аналогу тождества Фэя [14], [8]

$$
\phi(\hbar, z) \phi(\eta, w)=\phi(\hbar-\eta, z) \phi(\eta, z+w)+\phi(\eta-\hbar, w) \phi(\hbar, z+w),
$$

известному как ассоциативное уравнение Янга-Бакстера [15]:

$$
R_{a c}^{\hbar} R_{c b}^{\eta}=R_{a b}^{\eta} R_{a c}^{\hbar-\eta}+R_{c b}^{\eta-\hbar} R_{a b}^{\hbar}
$$

1) Обозначения для эллиптических функций в нашей работе совпадают с обозначениями из работ [9] и [10] (см. раздел 4). В упомянутых работах можно найти определение эллиптической $R$-матрицы, удовлетворяющей обсуждаемым здесь свойствам и тождествам. Некоторые важные определения и свойства будут приведены в ходе изложения.

2) Впервые идея рассматривать $R$-матрицу как обобщение скалярной функции была предложена в статье [13]. В этой работе использовалась стандартная нормировка $R_{12}^{\hbar} R_{21}^{\hbar}=1 \otimes 1$ для условия унитарности. 
Используя это уравнение вместе с условием унитарности (1.4) и антисимметричности ${ }^{3)}$

$$
R_{a b}^{\hbar}\left(z_{a}-z_{b}\right)=-R_{b a}^{-\hbar}\left(z_{b}-z_{a}\right),
$$

можно вывести [12], [10], что $R_{a b}^{\hbar}$ удовлетворяет уравнению Янга-Бакстера (1.1) и следующему кубическому тождеству:

$$
\begin{aligned}
& R_{12}^{\hbar} R_{23}^{\hbar} R_{31}^{\hbar}+R_{13}^{\hbar} R_{32}^{\hbar} R_{21}^{\hbar}=-N^{3} \wp^{\prime}(N \hbar) 1 \otimes 1 \otimes 1= \\
& \quad=N^{3}\left(\phi\left(N \hbar, z_{12}\right) \phi\left(N \hbar, z_{23}\right) \phi\left(N \hbar, z_{31}\right)+\phi\left(N \hbar, z_{13}\right) \phi\left(N \hbar, z_{32}\right) \phi\left(N \hbar, z_{21}\right)\right) 1 \otimes 1 \otimes 1,
\end{aligned}
$$

где $z_{a b}=z_{a}-z_{b}$. Некоторые важные приложения этого тождества обсуждаются в разделе 4 .

Целью работы является обобщение на старшие порядки по $R$ условия унитарности (1.4) и тождества (1.11). Основной результат составляет

Теорема 1. Пусть $R$-матрица удовлетворяет условию унитарности (1.4) и ассоциативному уравнению Янга-Бакстера (1.9). Тогда она также удовлетворяет следующему набору тождеств $n$-го порядка $\left(n \in \mathbb{Z}_{+}\right)$:

$$
\begin{aligned}
& \sum_{\substack{1 \leqslant i_{1}, \ldots, i_{n-1} \leqslant n, i_{c} \neq a, i_{b} \neq i_{c}}} R_{a i_{1}}^{\hbar} R_{i_{1} i_{2}}^{\hbar} \ldots R_{i_{n-2} i_{n-1}}^{\hbar} R_{i_{n-1} a}^{\hbar}= \\
& =\underbrace{1 \otimes \cdots \otimes 1}_{n} N^{n} \sum_{\substack{1 \leqslant i_{1}, \ldots, i_{n-1} \leqslant n, i_{c} \neq a, i_{b} \neq i_{c}}} \phi\left(N \hbar, z_{a}-z_{i_{1}}\right) \phi\left(N \hbar, z_{i_{1}}-z_{i_{2}}\right) \ldots \phi\left(N \hbar, z_{i_{n-1}}-z_{a}\right),
\end{aligned}
$$

где $а$ - биксированный индекс, $1 \leqslant a \leqslant n$. Для $n \geqslant 3$ тождества (1.12) можно nереписать в виде

$$
\sum_{\substack{1 \leqslant i_{1}, \ldots, i_{n-1} \leqslant n, i_{c} \neq a, i_{b} \neq i_{c}}} R_{a i_{1}}^{\hbar} R_{i_{1} i_{2}}^{\hbar} \ldots R_{i_{n-2} i_{n-1}}^{\hbar} R_{i_{n-1} a}^{\hbar}=\left.\underbrace{1 \otimes \cdots \otimes 1}_{n}(-N)^{n} \frac{d^{(n-2)} \wp(\eta)}{d \eta^{(n-2)}}\right|_{\eta=N \hbar} .
$$

Произведения $R$-матриц в суммах из $(1.12),(1.13)$ содержат все возможные комбинации попарно различных значений индексов из множества $\{1, \ldots, n\}$. Количество слагаемых в сумме равно $(n-1)$ !. Действительно, так как $a$ фиксировано, индекс $i_{1}$ может принимать $n-1$ возможное значение. После фиксации $i_{1}$ следующий индекс $i_{2}$ имеет $n-2$ возможных значений из множества $\{1, \ldots, n\} \backslash\left\{a, i_{1}\right\}$ и т. д. Следовательно, суммарное количество возможных наборов индексов равно $(n-1)$ !. Например, для $n=3$ равенство (1.13) воспроизводит (1.11), а для $n=4$ дает

$$
\begin{aligned}
R_{12}^{\hbar} R_{23}^{\hbar} R_{34}^{\hbar} R_{41}^{\hbar} & +R_{12}^{\hbar} R_{24}^{\hbar} R_{43}^{\hbar} R_{31}^{\hbar}+R_{13}^{\hbar} R_{32}^{\hbar} R_{24}^{\hbar} R_{41}^{\hbar}+R_{13}^{\hbar} R_{34}^{\hbar} R_{42}^{\hbar} R_{21}^{\hbar}+ \\
& +R_{14}^{\hbar} R_{42}^{\hbar} R_{23}^{\hbar} R_{31}^{\hbar}+R_{14}^{\hbar} R_{43}^{\hbar} R_{32}^{\hbar} R_{21}^{\hbar}=N^{4} \wp^{\prime \prime}(N \hbar) 1 \otimes 1 \otimes 1 \otimes 1 .
\end{aligned}
$$

\footnotetext{
3)Это свойство антисимметричности представляет собой матричный аналог свойства функции Кронекера $\phi(\eta, z)=-\phi(-\eta,-z)$. В работе [11] $R^{\hbar}(z)$ интерпретировалась как классическая $r$-матрица, свойство антисимметричности - как условие унитарности, а условие (1.4) не использовалось.
} 
Утверждение теоремы предполагает, конечно, что для $n \geqslant 3$ сумма произведений функций Кронекера в правой части тождества (1.12) равна $(-1)^{n} \wp^{(n-2)}(N \hbar)$. Скорее всего, это соотношение известно, однако найти его в литературе нам не удалось ${ }^{4)}$. В следующем разделе мы предлагаем простой способ вывода этих тождеств, который естественно обобщается на случай $R$-матричных тождеств. Их доказательство приведено в разделе 3. Аналог тождества (1.12) при $n=1$ обсуждается в разделе 4.

\section{2. СТАРШИЕ ТОЖДЕСТВА ДЛЯ ФУНКЦИЙ КРОНЕКЕРА}

В этом разделе мы доказываем

ПреДЛОЖЕНИЕ 1. Функиия Кронекера $\phi(\eta, z)$ удовлетворяет следующему набору тождеств п-го порядка для $n>2$ :

$$
\sum_{\substack{1 \leqslant i_{1}, \ldots, i_{n-1} \leqslant n, i_{c} \neq a, i_{b} \neq i_{c}}} \phi\left(\eta, z_{a}-z_{i_{1}}\right) \phi\left(\eta, z_{i_{1}}-z_{i_{2}}\right) \ldots \phi\left(\eta, z_{i_{n-1}}-z_{a}\right)=(-1)^{n} \frac{d^{(n-2)} \wp(\eta)}{d \eta^{(n-2)} .}
$$

Прежде чем перейти к доказательству, определим еще одну важную для наших целей функцию - первую функцию Эйзенштейна ${ }^{5)}$

$$
E_{1}(z)=\left\{\begin{array}{ll}
\frac{1}{z}, & \text { рациональный случай, } \\
\operatorname{cth} z, & \text { тригонометрический случай, } \\
\frac{\vartheta^{\prime}(z)}{\vartheta(z)}, & \text { эллиптический случай, }
\end{array} \quad E_{1}(-z)=-E_{1}(z) .\right.
$$

Она появляется в разложении функции Кронекера в окрестности полюса $\eta=0$,

$$
\phi(\eta, z)=\eta^{-1}+E_{1}(z)+\eta \frac{E_{1}^{2}(z)-\wp(z)}{2}+O\left(\eta^{2}\right)
$$

а также в формуле для производной

$$
\frac{d}{d \eta} \phi(\eta, z)=\left(E_{1}(\eta+z)-E_{1}(\eta)\right) \phi(\eta, z)
$$

и тождестве Фэя (1.8) для вырожденного случая $\eta=\hbar$ :

$$
\phi(\eta, z) \phi(\eta, w)=\phi(\eta, z+w)\left(E_{1}(\eta)+E_{1}(z)+E_{1}(w)-E_{1}(z+w+\eta)\right) .
$$

Используя равенства (2.4) и (2.5), получаем

$$
\frac{d}{d \eta} \phi(\eta, z) \equiv \phi^{\prime}(\eta, z)=\left(E_{1}(z+y)-E_{1}(y)\right) \phi(\eta, z)-\phi(\eta, z+y) \phi(\eta,-y) .
$$

Заметим, что это соотношение верно для любого $y \in \mathbb{C}$, тогда как его левая часть от $y$ не зависит.

4) Возможно, тождества такого типа можно вывести из формул для эллиптических определителей Коши, полученных Ройсенарсом [16].

5) Она простым образом связана с дзета-функцией Вейерштрасса: $E_{1}(z)=\zeta(z)+z \vartheta^{\prime \prime \prime}(0) / 3 \vartheta^{\prime}(0)$. 
Непосредственный способ доказательства тождеств типа (2.1) состоит в сравнении полюсов и вычетов левой и правой частей тождества. Легко убедиться, что левая часть равенства (2.1) является двоякопериодической функцией от $\eta$ и $z_{1}, \ldots, z_{n}$. Более того, можно проверить, что вычеты на диагоналях $z_{i}=z_{j}$ равны нулю. Это, однако, означает лишь, что левая часть есть двоякопериодическая функция от $\eta$ с особенностью типа $1 / \eta^{n}$ в окрестности $\eta=0$. Таким способом определить эту функцию однозначно сложно, т. е. сложно доказать отсутствие (в правой части) членов $c_{k}(\tau) \frac{d^{k} \wp(\eta)}{d \eta^{k}}$ с $k<n-2$. Вместо этого мы предлагаем другой способ доказательства тождества (2.1), основанный на соотношении (2.6).

ДокАЗАТЕЛЬСтво ПРЕДЛОЖЕНИЯ. Доказывать тождество (2.1) будем с помощью индукции по $n$. Для $n=3$ имеем хорошо известное равенство

$\phi\left(\eta, z_{1}-z_{2}\right) \phi\left(\eta, z_{2}-z_{3}\right) \phi\left(\eta, z_{3}-z_{1}\right)+\phi\left(\eta, z_{1}-z_{3}\right) \phi\left(\eta, z_{3}-z_{2}\right) \phi\left(\eta, z_{2}-z_{1}\right)=-\wp^{\prime}(\eta)$,

которое можно проверить непосредственно, сравнив полюсы и вычеты. Используя (2.5), а затем (1.7), его можно переписать в более узнаваемом виде

$$
E_{1}(\eta+z)+E_{1}(\eta-z)-2 E_{1}(\eta)=\frac{\wp^{\prime}(\eta)}{\wp(\eta)-\wp(z)}, \quad z=z_{a}-z_{b} .
$$

Пусть равенство (2.1) верно для $n$. Продифференцируем обе его части по $\eta$. Тогда правая часть примет вид

$$
-(-1)^{n+1} \frac{d^{n-1} \wp(\eta)}{d \eta^{n-1}}
$$

что совпадает с правой частью равенства (2.1) для $n:=n+1$, взятой с противоположным знаком. Для левой части получим

$$
\sum_{\substack{1 \leqslant i_{1}, \ldots, i_{n-1} \leqslant n, i_{c} \neq a, i_{b} \neq i_{c}}}\left(\phi_{a i_{1}}^{\prime}(\eta) \phi_{i_{1} i_{2}}(\eta) \ldots \phi_{i_{n-1} a}(\eta)+\cdots+\phi_{a i_{1}}(\eta) \phi_{i_{1} i_{2}}(\eta) \ldots \phi_{i_{n-1} a}^{\prime}(\eta)\right),
$$

где использованы обозначения

$$
\phi_{i j}(\eta)=\phi\left(\eta, z_{i}-z_{j}\right), \quad \phi_{i j}^{\prime}(\eta)=\frac{d}{d \eta} \phi\left(\eta, z_{i}-z_{j}\right) .
$$

Подставим в (2.8) формулу (2.6), выбирая каждый раз $y$ так, что для $\phi_{i j}^{\prime}(\eta)$ имеет место равенство $y=z_{j}-z_{n+1}$. Ответ для (2.8) после подстановки состоит из членов двух типов: вида $\phi^{n} E_{1}$ и вида $\phi^{n+1}$. Члены первого типа взаимно сокращаются для каждого выражения в скобках в (2.8) в силу свойства антисимметричности (2.2) функции $E_{1}$. Действительно, суммируя все такие слагаемые с фиксированными значениями индексов $i_{1}, \ldots, i_{n-1}$, получаем

$$
\begin{aligned}
0= & \phi_{a i_{1}}(\eta) \phi_{i_{1} i_{2}}(\eta) \ldots \phi_{i_{n-1} a}(\eta)\left(E_{1}\left(z_{a}-z_{i_{1}}+z_{i_{1}}-z_{n+1}\right)-E_{1}\left(z_{i_{1}}-z_{n+1}\right)+\right. \\
& +E_{1}\left(z_{i_{1}}-z_{i_{2}}+z_{i_{2}}-z_{n+1}\right)-E_{1}\left(z_{i_{2}}-z_{n+1}\right)+\cdots+ \\
& \left.+E_{1}\left(z_{i_{n-1}}-z_{a}+z_{a}-z_{n+1}\right)-E_{1}\left(z_{a}-z_{n+1}\right)\right) .
\end{aligned}
$$


Сумма всех членов второго типа (вида $\phi^{n+1}$ ) есть в точности левая часть равенства (2.1) для $n:=n+1$ с общим противоположным знаком:

$$
\begin{aligned}
& \sum_{\substack{1 \leqslant i_{1}, \ldots, i_{n-1} \leqslant n, i_{c} \neq a, i_{b} \neq i_{c}}}\left(\phi_{a i_{1}}^{\prime}(\eta) \phi_{i_{1} i_{2}}(\eta) \ldots \phi_{i_{n-1} a}(\eta)+\cdots+\phi_{a i_{1}}(\eta) \phi_{i_{1} i_{2}}(\eta) \ldots \phi_{i_{n-1} a}^{\prime}(\eta)\right)= \\
& =-\sum_{\substack{1 \leqslant i_{1}, \ldots, i_{n-1} \leqslant n, i_{c} \neq a, i_{b} \neq i_{c}}}\left(\phi_{a, n+1}(\eta) \phi_{n+1, i_{1}}(\eta) \phi_{i_{1} i_{2}}(\eta) \ldots \phi_{i_{n-1} a}(\eta)+\cdots+\right. \\
& \left.=-\sum_{\substack{1 \leqslant i_{1}, \ldots, i_{n-1} \leqslant n, i_{c} \neq a, i_{b} \neq i_{c}}} \phi_{a i_{1}}(\eta) \phi_{i_{1} i_{2}}(\eta) \ldots \phi_{i_{1} i_{2}}(\eta) \ldots \phi_{i_{n-1}, n+1}(\eta) \phi_{n+1, a}(\eta)\right)=
\end{aligned}
$$

В справедливости последнего равенства легко убедиться, рассмотрев структуру индексов суммирования. Значения всех индексов в каждом члене различны и отличны от "внешнего" индекса ${ }^{6)} a$. Такое же выражение получается и из членов второго типа после подстановки. В то же время количество слагаемых второго типа в (2.8) (и, следовательно, в (2.11)) равно $n$ ! (до дифференцирования по $\eta$ было $(n-1)$ ! слагаемых ${ }^{7)}$, а далее к каждому из них применялось правило Лейбница), что совпадает с числом слагаемых в $(2.1)$ для $n:=n+1$.

Сравнение выражений (2.11) и (2.7) завершает доказательство предложения.

\section{3. СТАРШИЕ ТОЖДЕСТВА ДЛЯ $R$-МАТРИЦ}

В этом разделе мы докажем теорему 1. Доказательство аналогично приведенному выше доказательству предложения 1.

$R$-матричным аналогом первой функции Эйзенштейна (2.2) является классическая $r$-матрица $r_{a b}(z)$. Она появляется в классическом пределе так же, как и функция $E_{1}$ в $(2.3)$ :

$$
R_{12}^{\hbar}(z)=\frac{1}{\hbar} 1 \otimes 1+r_{12}(z)+\hbar m_{12}(z)+O\left(\hbar^{2}\right)
$$

Аналогично функции $E_{1}$ классическая $r$-матрица кососимметрична ${ }^{8)}$ :

$$
r_{a b}\left(z_{a}-z_{b}\right)=-r_{b a}\left(z_{b}-z_{a}\right), \quad m_{a b}=m_{b a}=\frac{1}{2}\left(r_{a b}^{2}-1 \otimes 1 N^{2} \wp\left(z_{a}-z_{b}\right)\right) .
$$

$R$-матричный аналог равенства (2.6) следует из ассоциативного уравнения Янга-Бакстера (1.9) и равенства (3.1) в пределе $\eta \rightarrow \hbar$ :

$$
\partial_{\hbar} R_{a b}^{\hbar} \equiv J_{a b}^{\hbar}=R_{a b}^{\hbar} r_{a c}+r_{c b} R_{a b}^{\hbar}-R_{a c}^{\hbar} R_{c b}^{\hbar} .
$$

ДоказАТеЛЬство теоремы 1. Равенство (1.12) при $n=2$ есть условие унитарности (1.4), равенство (1.12) при $n=3$ - это известное из работ [12], [10] тождество (1.11), а для $n=1$ это равенство выполнено в смысле соотношения (4.1), выписанного ниже.

\footnotetext{
6) Значение "внешнего" индекса а на самом деле несущественно (см. ниже замечание 1).

7) См. комментарий после формулы (1.13).

8)Это является следствием как унитарности (в $\hbar^{-1}-, \hbar^{0}$-порядках разложения), так и условия (1.10) (в $\hbar^{0}-, \hbar^{1}$-порядках разложения). Матрица $r_{12}(z)$ из (3.1) - это классическая $r$-матрица Белавина-Дринфельда [17].
} 
Докажем равенство (1.13). Тогда (1.12) следует из скалярного аналога (1.13) предложения 1. Идея доказательства воспроизводит ход рассуждений для (2.1).

Доказывать равенство (1.13) будем с помощью индукции по $n$. Пусть оно верно для $n$. Умножим (тензорно) обе части равенства (1.13) на 1 (здесь 1 - единичная матрица размера $N \times N)$ и продифференцируем их по $\hbar$. Правая часть примет вид

$$
-\left.\underbrace{1 \otimes \cdots \otimes 1}_{n+1}(-N)^{n+1} \frac{d^{(n-1)} \wp(\eta)}{d \eta^{(n-1)}}\right|_{\eta=N \hbar},
$$

что совпадает с правой частью равенства (1.13), взятой при $n:=n+1$ с противоположным знаком.

Левая часть принимает вид

$$
\sum_{\substack{1 \leqslant i_{1}, \ldots, i_{n-1} \leqslant n, i_{c} \neq a, i_{b} \neq i_{c}}}\left(J_{a i_{1}}^{\hbar} R_{i_{1} i_{2}}^{\hbar} \ldots R_{i_{n-2} i_{n-1}}^{\hbar} R_{i_{n-1} a}^{\hbar}+\cdots+R_{a i_{1}}^{\hbar} R_{i_{1} i_{2}}^{\hbar} \ldots R_{i_{n-2} i_{n-1}}^{\hbar} J_{i_{n-1} a}^{\hbar}\right) .
$$

Это выражение аналогично (2.8). Как и в скалярном случае, подставляя $J_{a b}^{\hbar}$ из (3.3) при $c=n+1$, получаем левую часть равенства (1.13), взятую для $n:=n+1$ с общим противоположным знаком. Ответ получается из членов вида $-R_{i, n+1}^{\hbar} R_{n+1, j}$ в (3.3).

Прокомментируем сокращение членов, содержащих классические $r$-матрицы $r_{a b}$. Вклад от таких членов в выражение в скобках в (3.5) равен

$$
\begin{aligned}
& \left(R_{a i_{1}}^{\hbar} r_{a, n+1}+r_{n+1, i_{1}} R_{a i_{1}}^{\hbar}\right) R_{i_{1} i_{2}}^{\hbar} \ldots R_{i_{n-1} a}^{\hbar}+ \\
& \quad+R_{a i_{1}}^{\hbar}\left(R_{i_{1} i_{2}}^{\hbar} r_{i_{1}, n+1}+r_{n+1, i_{2}} R_{i_{1} i_{2}}^{\hbar}\right) R_{i_{2} i_{3}}^{\hbar} \ldots R_{i_{n-1} a}^{\hbar}+\cdots+ \\
& \quad+R_{a i_{1}}^{\hbar} R_{i_{1} i_{2}}^{\hbar} \ldots R_{i_{n-2} i_{n-1}}^{\hbar}\left(R_{i_{n-1} a}^{\hbar} r_{i_{n-1}, n+1}+r_{n+1, a} R_{i_{n-1} a}^{\hbar}\right)
\end{aligned}
$$

Первое и последнее слагаемые (и только они) содержат $r_{a, n+1}$. Эти слагаемые сокращаются, так как $r_{a, n+1}$ можно передвинуть в первом члене ${ }^{9)}$ на предпоследнее место, а также в силу свойства антисимметричности (3.2). Все оставшиеся члены собираются в коммутатор

$$
\left[r_{n+1, i_{1}}+r_{n+1, i_{2}}+\cdots+r_{n+1, i_{n-1}}, R_{a i_{1}}^{\hbar} R_{i_{1} i_{2}}^{\hbar} \ldots R_{i_{n-1} a}^{\hbar}\right]
$$

в силу того, что все $r$-матрицы вида $r_{n+1, k}$ в своем произведении можно передвинуть влево, а $r$-матрицы с индексами $r_{k, n+1}$ - вправо. Этот коммутатор равен нулю, так как произведение $R$-матриц является скалярным оператором по предложению индукции. Таким образом, выражение (3.6) также равно нулю. Теорема доказана.

\section{4. НЕКОТОРЫЕ ЗАМЕЧАНИЯ И ФОРМУЛЫ}

ЗАмечАниЕ 1 . Группа перестановок набора $\left(z_{1}, \ldots, z_{n}\right)$ преобразует $R$-матричные тождества (1.12), (1.13) с некоторым "внешним" индексом $a$ в тождество того же вида для другого $\tilde{a} \neq a$. Для этого нужно переобозначить $\left\{z_{k}\right\}$ и подействовать на (1.12), (1.13) произведением соответствующих операторов перестановки (с обеих сторон). В скалярном случае тождество (2.1) инвариантно относительно действия

${ }^{9)}$ Произведение $R_{i_{1} i_{2}}^{\hbar} \ldots R_{i_{n-2}, i_{n-1}}^{\hbar}$ не содержит индексов $a$ и $n+1$ и, следовательно, коммутирует с $r_{a, n+1}$. 
группы перестановок: его левая часть в действительности не зависит от индекса $a$, так как функции $\phi$ в произведениях можно переупорядочить так, что (2.1) примет вид исходного тождества для другого индекса $\tilde{a} \neq a$.

Например, для $n=3$, очевидно, имеем

$$
\begin{aligned}
& \phi\left(\eta, z_{1}-z_{2}\right) \phi\left(\eta, z_{2}-z_{3}\right) \phi\left(\eta, z_{3}-z_{1}\right)+\phi\left(\eta, z_{1}-z_{3}\right) \phi\left(\eta, z_{3}-z_{2}\right) \phi\left(\eta, z_{2}-z_{1}\right)= \\
& \quad=\phi\left(\eta, z_{2}-z_{1}\right) \phi\left(\eta, z_{1}-z_{3}\right) \phi\left(\eta, z_{3}-z_{2}\right)+\phi\left(\eta, z_{2}-z_{3}\right) \phi\left(\eta, z_{3}-z_{1}\right) \phi\left(\eta, z_{1}-z_{2}\right) .
\end{aligned}
$$

Здесь $a=1$ и $\tilde{a}=2$. Это соотношение справедливо и для произвольных $n, a, \tilde{a}$.

ЗАмечание 2. $R$-матричное тождество (1.12) имеет смысл и в случае $n=1$ : для $R_{a b}^{\hbar}(z)$ можно отождествить $a$-ю и $b$-ю компоненты тензорного произведения, сохранив условие $z \neq 0$. Тогда

$$
R_{a a}^{\hbar}(z)=1_{a} N \phi\left(N \hbar, \frac{z}{N}\right)
$$

где $1_{a}$ - единичная матрица размера $N \times N$, стоящая в качестве $a$-й компоненты Для доказательства равенства (4.1) рассмотрим наиболее общий случай - эллиптическую $R$-матрицу Белавина [6]. В наших обозначениях и с нашей нормировкой она имеет вид

$$
R_{12}^{\hbar}(z)=\sum_{\alpha \in \mathbb{Z}_{N} \times \mathbb{Z}_{N}} \varphi_{\alpha}\left(z, \omega_{\alpha}+\hbar\right) T_{\alpha} \otimes T_{-\alpha} \in \operatorname{Mat}(N, \mathbb{C})^{\otimes 2},
$$

где

$$
\varphi_{\alpha}\left(z, \omega_{\alpha}+\hbar\right)=\exp \left(2 \pi \imath \frac{\alpha_{2}}{N} z\right) \phi\left(z, \omega_{\alpha}+\hbar\right), \quad \omega_{\alpha}=\frac{\alpha_{1}+\alpha_{2} \tau}{N},
$$

a $\left\{T_{\alpha}\right\}-$ специальный базис в пространстве $\operatorname{Mat}(N, \mathbb{C})$ :

$$
T_{\alpha} T_{\beta}=\kappa_{\alpha, \beta} T_{\alpha+\beta}, \quad \kappa_{\alpha, \beta}=\exp \left(\frac{\pi \imath}{N}\left(\beta_{1} \alpha_{2}-\beta_{2} \alpha_{1}\right)\right),
$$

другими словами,

$$
T_{\alpha} T_{-\alpha}=1_{N \times N}
$$

(подробнее см. приложение в работе [10]). Из соотношений (4.2) и (4.5) следует, что

$$
R_{11}^{\hbar}(z)=1_{N \times N} \sum_{\alpha \in \mathbb{Z}_{N} \times \mathbb{Z}_{N}} \varphi_{\alpha}\left(z, \omega_{\alpha}+\hbar\right) .
$$

Сумма в левой части данного равенства равна $N \phi(N \hbar, z / N)$. Это частный случай конечного преобразования Фурье функции Кронекера [9].

ЗАмечАниЕ 3 . Квантовые $R$-матрицы используются для построения классических пар Лакса в системах типа Калоджеро [12]. В $g l_{n}$-случае такая матрица Лакса имеет вид

$$
\mathcal{L}(\hbar)=\sum_{a, b=1}^{n} \widetilde{\mathrm{E}}_{a b} \otimes \mathcal{L}_{a b}(\hbar), \quad \mathcal{L}_{a b}(\hbar)=\delta_{a b} p_{a} 1_{a} \otimes 1_{b}+\nu\left(1-\delta_{a b}\right) R_{a b}^{\hbar}\left(z_{a}-z_{b}\right),
$$

где $\widetilde{\mathrm{E}}_{a b}$ - стандартный базис в пространстве $\operatorname{Mat}(n, \mathbb{C})$, набор $R$-матричных спектральных параметров $\left\{z_{a}\right\}-$ координаты калоджеровских частиц, $p_{a}-$ импульсы 
частиц, а $\hbar$ играет роль спектрального параметра. Формула (4.7) является обобщением формулы Кричевера [18].

В работе [12] было сделано следующее предположение: диагональные элементы (блоки) матрицы $\operatorname{tr} \mathcal{L}^{k}(\hbar)$ суть скалярные операторы с коэффициентами, которые равны этому же элементу, вычисленному для матрицы $\operatorname{tr} l^{k}(\hbar)$ - (обычной) кричеверовской матрицы Лакса $l_{a b}(\hbar)=p_{a} \delta_{a b}+\nu\left(1-\delta_{a b}\right) N \phi\left(N \hbar, z_{a}-z_{b}\right)$. Для $n=2$ это утверждение следует из условия унитарности (1.4), а для $n=3$ - из кубического тождества (1.11). Утверждение теоремы 1, очевидно, относится к тому же типу. В действительности тождество (1.12) можно рассматривать как часть упомянутого предположения для $k=n$.

ЗАмечАниЕ 4. Из квантового уравнения Янга-Бакстера (1.1) и кубического тождества (1.11) следует, что

$$
\left[r_{a b}, m_{a c}+m_{b c}\right]+\left[r_{a c}, m_{a b}+m_{b c}\right]=0
$$

где $r_{a b}$ и $m_{a b}$ определены через классический предел (3.1) (см. детали в работе [12]). Это уравнение гарантирует коммутативность различных связностей Книжника-Замолодчикова-Бернара ${ }^{10)}$

$$
\begin{aligned}
& \nabla_{a}=\partial_{z_{a}}+\sum_{b: b \neq a} r_{a b}\left(z_{a}-z_{b}\right), \quad a=1, \ldots, n, \\
& \nabla_{\tau}=\partial_{\tau}+\sum_{b>c} m_{b c}\left(z_{b}-z_{c}\right)
\end{aligned}
$$

а именно $\left[\nabla_{a}, \nabla_{\tau}\right]=0$ (равенство $\left[\nabla_{a}, \nabla_{b}\right]=0$ следует из классического уравнения Янга-Бакстера). Полученные тождества (1.12), (1.13) дают набор уравнений для $r_{a b}, m_{a b}$ и старшие члены разложения (3.1). Простейшим примером является коэффициент при $1 / \hbar^{n-2}$ в (1.13):

$$
\sum_{c<a<b}\left[r_{c a}, r_{a b}\right]_{+}+\left[r_{a b}, r_{b c}\right]_{+}+\left[r_{b c}, r_{c a}\right]_{+}=-(n-2) \sum_{b \neq c} m_{b c}
$$

\section{Список литературы}

[1] C. N. Yang, Phys. Rev. Lett., 19:23 (1967), 1312-1315.

[2] И. В. Чередник, ТМФ, 43:1 (1980), 117-119.

[3] A. Smirnov, Central Eur. J. Phys., 8:4 (2010), 542-554, arXiv: 0903.1466.

[4] A. Levin, M. Olshanetsky, A. Zotov, JHEP, 07 (2014), 012, 38 pp., arXiv: 1405.7523; G. Aminov, S. Arthamonov, A. Smirnov, A. Zotov, J. Phys. A: Math. Theor., 47:30 (2014), 305207, 19 pp., arXiv: 1402.3189.

[5] A. Antonov, K. Hasegawa, A. Zabrodin, Nucl. Phys. B, 503:3 (1997), 747-770, arXiv: hep-th/9704074.

[6] R. J. Baxter, Ann. Phys., 70:1 (1972), 193-228.

[7] A. A. Belavin, Nucl. Phys. B, 180:2 (1981), 189-200.

[8] А. Вейль, Эллиптические функиии по Эйзенштейну и Кронекеру, Мир, М., 1978.

10) Тождества (4.8) можно получить также из вырожденного ассоциативного уравнения Янга-Бакстера (3.3). 
[9] А. М. Левин, М.А. Ольшанецкий, А. В. Зотов, ТМФ, 184:1 (2015), 41-56, arXiv: 1501.07351.

[10] A. Levin, M. Olshanetsky, A. Zotov, J. Phys. A: Math. Theor., 49:1 (2016), 014003, 19 pp., arXiv: 1507.02617.

[11] A. Polishchuk, Adv. Math., 168:1 (2002), 56-95.

[12] A. Levin, M. Olshanetsky, A. Zotov, JHEP, 10 (2014), 109, 28 pp., arXiv: 1408.6246.

[13] V. V. Bazhanov, Yu. G. Stroganov, "On connection between the solutions of the quantum and classical triangle equations", Труды международного семинара по проблемам физики высоких энергий и квантовой теории поля. Т. 1 (Протвино, июль 1983 г.), Ин-т физики высоких энергий, Протвино, 1983, 51-53; Л. А. Тахтаджян, Зап. научн. сем. ЛОМИ, 133 (1984), 258-276.

[14] J. D. Fay, Theta Functions on Riemann Surfaces, Lecture Notes in Mathematics, 352, Springer, Berlin, 1973; Д. Мамфорд, Лекиии о тэта-функииях, Мир, М., 1988.

[15] S. Fomin, A. N. Kirillov, Discrete Math., 153:1-3 (1996), 123-143.

[16] S. N. M. Ruijsenaars, Commun. Math. Phys., 110:2 (1987), 191-213.

[17] А. А. Белавин, В. Г. Дринфельд, Функи. анализ и его прил., 16:3 (1982), 1-29.

[18] И. М. Кричевер, Функи. анализ и его прил., 14:4 (1980), 45-54.

Поступила в редакцию 8.11.2015, после доработки 18.12.2015 